\title{
Electropolymerization of Phenol on Carbon Steel and Stainless Steel Electrodes in Carbonate Aqueous Medium
}

\author{
Pedro Garcés, ${ }^{*}$ Rocío LAPUente, ${ }^{* *}$ Luís García Andión, ${ }^{*}$ Francisco Cases, ${ }^{* * *}$ \\ Emilia MORAllón, ${ }^{* *}$ and José Luís VÁZQUez ${ }^{* *, \dagger}$ \\ *Departamento de Ingeniería de la Construcción, O.P., Inf. Urbana, Universidad de Alicante. Apdo. 99. 03080-Alicante, Spain \\ ${ }^{* *}$ Departamento de Química-Física. Universidad de Alicante. Apdo. 99. 03080-Alicante, Spain \\ *** Departamento de Ingeniería Textily Papelera, EPS de Alcoy, Universidad Politécnica de Valencia. Paseo del Viaducto, 1.03800-Alcoy, \\ Spain
}

(Received November 8, 1999)

\begin{abstract}
Cyclic voltammetry, chronoamperometry and FTIR-ATR techniques were used to investigate the phenol electropolymerization on carbon steel and stainless steel electrodes in carbonate aqueous medium. Phenol electropolymerization occurs on a passivated surface and the polymer grows to thickness of 0.1 to $0.2 \mu \mathrm{m}$. Polymeric films are adherent and stables under ambient conditions. The polymeric film maintains aromatic character and contains etherlinked rings.
\end{abstract}

KEY WORDS Carbon Steel / Stainless Steel / Phenol / Electropolymerization / Reflection Spectroscopy /

The preparation of organic polymer coatings on different metallic substrates is of great interest in the field of material protection. For this reason, the study of the formation of polymeric films on oxidizable metals by electropolymerization in aqueous media has been widely studied in recent years, with attention mainly to electron-conducting polymers obtained by electroxidation of aniline, pyrrole, pyridine, thiophene, etc., and substituted derivatives. The properties of these polymeric coatings depend largely on the electropolymerization conditions: solvent, electrolyte, $\mathrm{pH}$, monomer concentration, metallic substrate nature, electrochemical treatment, etc.

Phenol and its substituted derivatives can be electropolymerized by oxidation in aqueous and non-aqueous solutions giving phenol polymeric films..$^{1-21}$ In general, these films are very thin, adherent and present low water mobility and low permeability to different ionic and molecular species. $\mathrm{pH}$ influences the electropolymerization mechanism of phenol on Pt electrodes. C-C bonds are mainly formed in acid medium ${ }^{5}$ whereas $\mathrm{C}-\mathrm{O}$ bonds are mainly formed in alkaline medium. ${ }^{10,15}$

The electrodeposition of conducting polymers on oxidizable metals is not easy, because the metal dissolves before the electropolymerization potential of the monomer is reached. Thus, it is necessary to find electrochemical conditions, to produce partial passivation and decrease in the metal dissolution rate without preventing electropolymerization. In 11, 14, 21 the authors used different amines in the formation of polyoxyphenylene coatings on $\mathrm{Fe}$ electrodes in aqueous-alcoholic and alcoholic alkaline solutions. The amines are adsorbed on $\mathrm{Fe}$ electrodes inhibiting the metal oxidation. However, other electrode reactions such as monomer oxidation are not avoided. When applied potential is high the amine is desorbed and the inhibition of metal oxidation is not produced. ${ }^{11}$ The amines were used in alkaline methanol medium to get passivating films onto mild steel from monomers that are oxidized at higher potentials than Fe. Poor coating is obtained in amine free methanol solutions. ${ }^{3,21}$ Therefore, it is assumed that the role of amines is preventing the formation of metal oxide on the electrode surface.

The presence of inorganic layers on Fe surface electrode does not necessarily prevent the electropolymerization of phenol. Even at potentials where the Fe electrode dissolves strongly, it is still possible to obtain a polymer coating if the medium allows the formation of a passivating layer on the Fe surface. ${ }^{11,18}$

The use of alkaline solutions in the electropolymerization of phenol on carbon steel and stainless steel electrodes helps to obtain stable passive layers that significantly allow the formation of polymeric films on the electrode surface. Film thickness and permeability of polymeric films obtained on platinum electrodes in carbonate medium or $\mathrm{NaOH}$ medium are different. Polymeric film thickness is smaller and more permeable in relation with oxide surface formation, $\mathrm{O}_{2}$ evolution, hydrogen adsorption-desorption process, etc., when is created in $\mathrm{NaOH}$ medium. ${ }^{22}$

This work demonstrates the formation of effective phenol polymeric films on carbon steel and stainless steel by electropolymerization of phenol monomer in aqueous carbonate solutions. The characteristics of the polymeric films formed were studied by cyclic voltammetry, Potentiostatic Current Transients and FTIR-ATR techniques. Scanning Electron Microscopy (SEM) was used to study film morphology.

\section{EXPERIMENTAL}

The test solution was $0.1 \mathrm{M} \mathrm{Na}_{2} \mathrm{CO}_{3}$ from Merck p.a. Phenol solution was prepared from Merck p.a. reagents. Water was obtained from a Millipore-Milli-Q System with a resistivity near to $18.2 \mathrm{M} \Omega \mathrm{cm}$. All potentials refer to the reversible hydrogen electrode $(\mathrm{RHE})$ immersed in the same test solution.

The electrode materials used in this work were: car-

${ }^{\dagger}$ To whom correspondence should be addressed (Tel: +34-965-90-35-36, Fax: +34-965-90-35-37, E-mail: jl.vazquez@ua.es). 


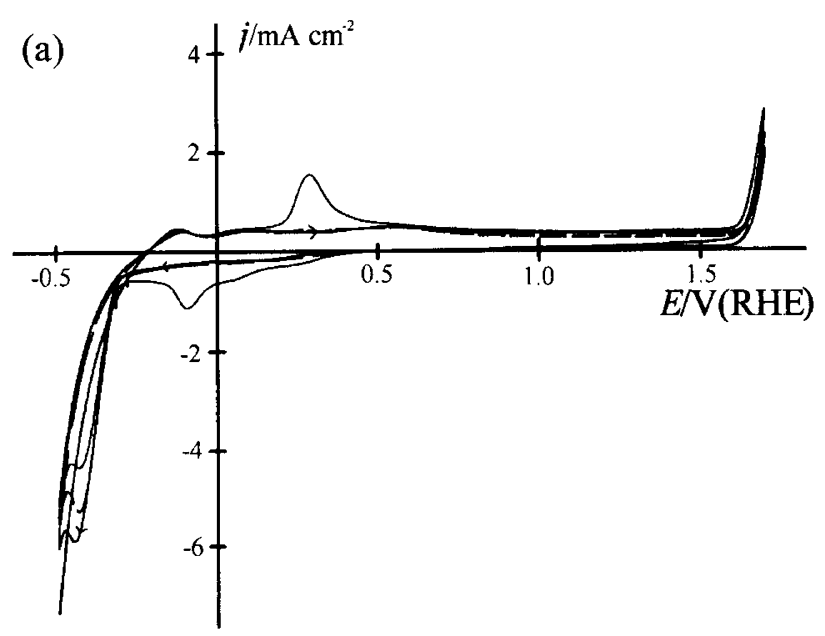

(b)

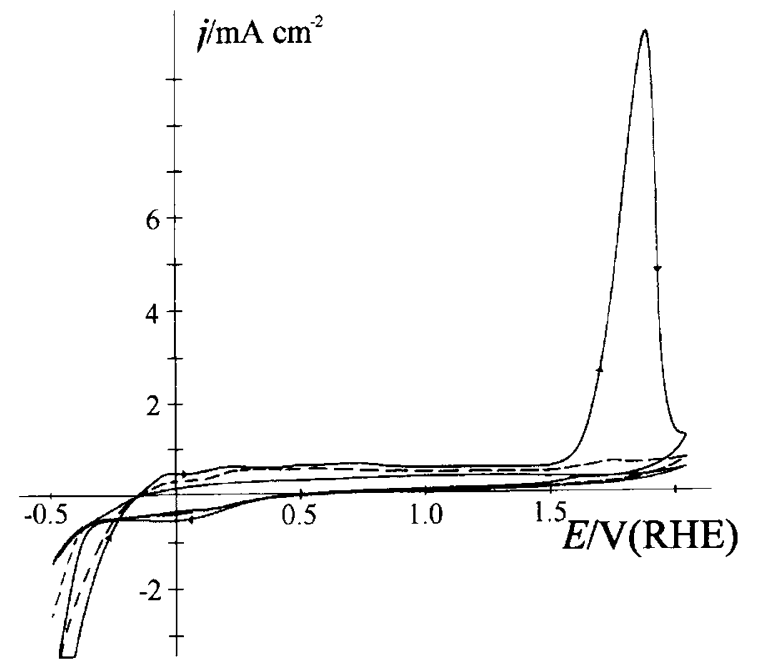

(c)

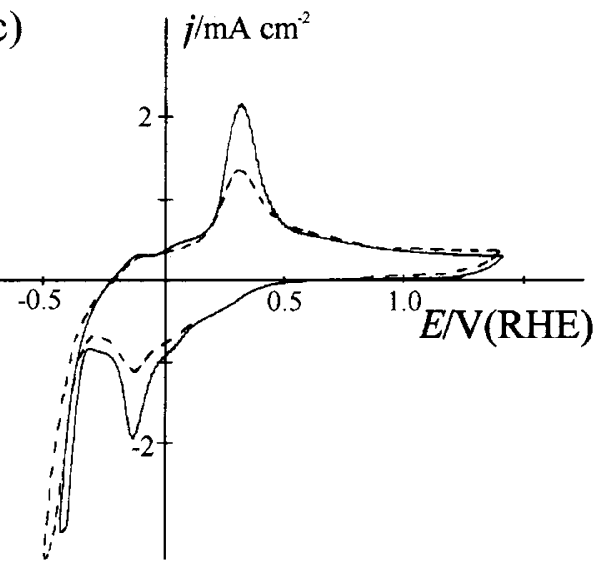

Figure 1. Cyclic voltammograms for CS electrode immersed in: (a) $0.1 \mathrm{M} \mathrm{Na}_{2} \mathrm{CO}_{3}$ solution. ( - ) first, ( - - ) second and (-) fifth cycle up to $1.7 \mathrm{~V}$. (b) $0.1 \mathrm{M} \mathrm{Na}_{2} \mathrm{CO}_{3}+6 \times 10^{-2} \mathrm{M}$ phenol solution. $(\longrightarrow)$ first, ( - - ) second and $(\longrightarrow)$ fifth cycle up to $2.05 \mathrm{~V}$.(c) $0.1 \mathrm{M} \mathrm{Na}_{2} \mathrm{CO}_{3}$. ( - - - ) covered of polymeric film and($\longrightarrow$ ) clean of polymeric film. Tenth cycle up to $1.4 \mathrm{~V} . \mathrm{v}=50 \mathrm{mV} \mathrm{s}^{-1}$.

bon steel (CS) (chemical composition in wt\%: C 0.160 , $M_{n} 0.600$, Si $0.160, \mathrm{~S} 0.031, \mathrm{P} 0.023, \mathrm{Cr} 0.130$, Ni 0.180 , Mo $0.050, \mathrm{~N} \mathrm{0.009,} \mathrm{Cu} 0.471)$ and stainless steel (SS) (chemical composition in wt\%: $\mathrm{C} \leq 0.050, \mathrm{Si} \leq 0.750, M_{n}$ $\leq 2.000$, P 0.040 , S 0.015 , Cr 18-19, Ni 8.5-9). In both cases cylindrical electrodes with 6 and $8 \mathrm{~mm}$ diameter, respectively, were employed.

CS and SS electrodes were degreased with acetone. Before each electrochemical experiment, they were polished with alumina of $0.05 \mu \mathrm{m}$ grade (Buehler) and after that, cleaned with ultrapure water in an ultrasonic bath. To remove surface oxides, the electrodes were cathodically polarized in the test solution at a potential of -0.5 $\mathrm{V}$ for $5 \mathrm{~min}$. For electropolymerization, the electrode treated as above was immersed in carbonate + phenol solution and polarization was started.

Fourier Transform Infrared Attenuated Total Reflection (FTIR-ATR) experiments were performed with a Nicolet Magna 550 Spectrometer equipped with a DTGS detector and a $45^{\circ} \mathrm{KRS}-5$ ATR crystal. Spectra were collected with a resolution of $8 \mathrm{~cm}^{-1}$.

A Jeol JSM-840 SEM was used to observe surface morphology.

\section{RESULTS AND DISCUSSION}

\section{Electrochemical Results}

Figure 1a shows voltammetric response obtained with a carbon steel electrode in $0.1 \mathrm{M} \mathrm{Na}_{2} \mathrm{CO}_{3}$ solution. During the first positive scan up to $0.6 \mathrm{~V}$, the electrode shows an active state in which its surface is oxidized. At potentials higher than $0.6 \mathrm{~V}$, passivation of the electrode surface occurs and at about $1.6 \mathrm{~V}$ oxygen evolution starts. The voltammetric profile changes with the number of sweeps in this range of potential and well-defined anodic and cathodic peaks appear.

Figure 1b shows voltammetric response of carbon steel electrode in presence of $6 \times 10^{-2} \mathrm{M}$ phenol in the $0.1 \mathrm{M}$ $\mathrm{Na}_{2} \mathrm{CO}_{3}$ solution. During the first positive scan up to 1.5 $\mathrm{V}$ the voltammetric profile is roughly the same as in Figure 1a (phenol free solution). From this potential, a sharp oxidation peak is obtained with a maximum approximately at $1.9 \mathrm{~V}$, associated with the oxidation of phenol. In following sweeps, the peak disappears and phenol oxidation is practically inhibited. Evolution of the voltammetric profile with the number of sweeps is different from that observed in Figure 1a, as may be concluded from the following:

i) Anodic and cathodic peaks that appear between -0.2 and $0.6 \mathrm{~V}$ in Figure 1a associated with the oxidation of the CS and reduction of $\mathrm{Fe}$ (III) species are not observed in presence of phenol in the solution.

ii) The current density in the overall potential range diminishes.

iii) Oxygen evolution shifts to more positive potentials (about $400 \mathrm{mV}$ ).

The oxidation of phenol on platinum electrodes in carbonate medium is inhibited by the formation of a passivating film. This film also avoids the formation of surface oxides on platinum electrodes. ${ }^{15}$ Therefore, the voltammetric behavior in Figure $1 \mathrm{~b}$ in the presence of phenol in solution could be associated with the formation of a low permeable polymeric film on the carbon steel surface.

To confirm the existence of a polymeric film on the electrode surface, the covered electrode, thoroughly 


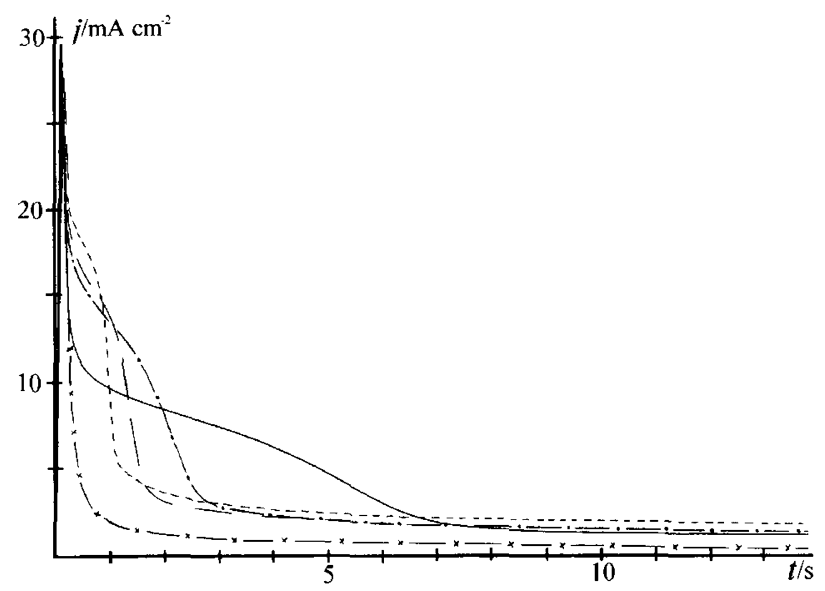

Figure 2. Current transients obtained with CS electrode in 0.1 $\mathrm{M} \mathrm{Na} \mathrm{CO}_{3}+0.1 \mathrm{M}$ phenol solution at different potential limits: 1.6 $\mathrm{V}(-\mathrm{X}-), 1.9 \mathrm{~V}\left(-\frac{1}{\longrightarrow}, 1.95 \mathrm{~V}(-\cdot-), 2.0 \mathrm{~V}(--)\right.$, and $2.1 \mathrm{~V}$ $(-\cdots)$.

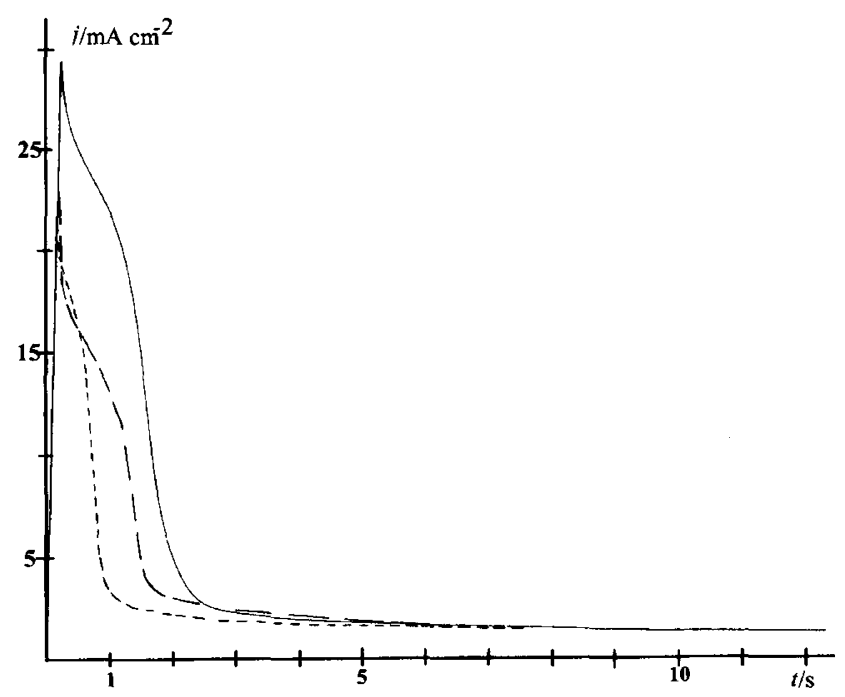

Figure 3. Current transients obtained with CS electrode in 0.1 $\mathrm{M} \mathrm{Na} \mathrm{CO}_{3}$ with different phenol concentrations. Potential limit constant of $2.0 \mathrm{~V}:(-\cdots) 6 \times 10^{-2} \mathrm{M},(---) 0.1 \mathrm{M}$, and $(-\square)$ $0.2 \mathrm{M}$.

Table I. Passivation time and charge for different potential limits and different phenol concentrations for CS electrodes

\begin{tabular}{|c|c|c|c|c|c|c|}
\hline [Phenol] / M & \multicolumn{3}{|c|}{ Passivation time $/ \mathrm{s}^{\mathrm{a}}$} & \multicolumn{3}{|c|}{ Passivation charge $/ \mathrm{mC} \mathrm{cm}^{-2 b}$} \\
\hline \multirow{2}{*}{0.06} & $1.95 \mathrm{~V}$ & $2.0 \mathrm{~V}$ & $2.1 \mathrm{~V}$ & $1.95 \mathrm{~V}$ & $2.0 \mathrm{~V}$ & $2.1 \mathrm{~V}$ \\
\hline & 1.8 & 1.2 & 1.2 & 19.3 & 16.3 & 15.9 \\
\hline 0.1 & 2.7 & 1.8 & 1.5 & 34.6 & 23.8 & 22.7 \\
\hline 0.2 & 6.8 & 2.4 & 2.0 & 57.6 & 40.5 & 37.1 \\
\hline
\end{tabular}

${ }^{\mathrm{a}}$ Passivation time corresponds to time where stationary current value starts. ${ }^{\mathrm{b}}$ Obtained by integration between $t=0$ and passivation time.

Table II. Passivation time and charge for different potential limits and different phenol concentrations for SS electrodes

\begin{tabular}{|c|c|c|c|c|c|c|}
\hline [Phenol] / M & \multicolumn{3}{|c|}{ Passivation time $/ \mathrm{s}^{\mathrm{a}}$} & \multicolumn{3}{|c|}{ Passivation charge $/ \mathrm{mC} \mathrm{cm}-2 \mathrm{~b}$} \\
\hline \multirow{2}{*}{0.06} & $1.90 \mathrm{~V}$ & $1.95 \mathrm{~V}$ & $2.0 \mathrm{~V}$ & $1.90 \mathrm{~V}$ & $1.95 \mathrm{~V}$ & $2.0 \mathrm{~V}$ \\
\hline & 1.4 & 1.2 & 1.2 & 12.7 & 14.6 & 13.9 \\
\hline 0.1 & 2.8 & 1.8 & 1.3 & 28.4 & 20.8 & 19.3 \\
\hline 0.2 & 6.8 & 2.8 & 1.6 & 63.1 & 34.0 & 24.6 \\
\hline
\end{tabular}

${ }^{\mathrm{a}}$ Passivation time corresponds to time where stationary current value starts. ${ }^{\mathrm{b}}$ Obtained by integration between $t=0$ and passivation time.

washed with ultrapure water, was immersed in a $0.1 \mathrm{M}$ $\mathrm{Na}_{2} \mathrm{CO}_{3}$ solution free of phenol and cycled between -0.5 and $1.4 \mathrm{~V}$. Figure $1 \mathrm{c}$ shows the tenth cycle for bare and coated electrodes. Evolution of the voltammetric profile with the number of sweeps is slower when the polymeric film exists on the electrode surface. Thus, the peak current associated with dissolution of $\mathrm{CS}$ electrode is smaller (about 40\% less) for the covered electrode than for clean CS electrode. The polymer film obtained from phenol oxidation thus seems to have a protective effect against the oxidation of the electrode surface.

The formation of polymer film due to phenol oxidation is observed when SS electrode is used. The voltammogram obtained with SS electrode in presence of phenol in the solution is very similar to the one obtained on CS. The oxidation of phenol gives one peak at approximately $1.85 \mathrm{~V}$ and is inhibited in the second cycle with the formation of a polymeric film on the electrode surface. The peak current associated with SS oxidation in a $0.1 \mathrm{M} \mathrm{Na}_{2}$ $\mathrm{CO}_{3}$ solution also decreases about $40 \%$ when the electrode surface is covered by the polymeric film.

The electrochemical behavior of the coated electrodes in carbonate solution free of phenol is not affected by the method of coating synthesis, applying a constant potential $(1.9 \mathrm{~V})$ or sweeping between -0.5 and $2.0 \mathrm{~V}$.

The different polymeric films created on both steel electrodes are adherent and stable under ambient conditions.

\section{Potentiostatic Current Transient}

The polymer formation was studied by potential steps. Carbon and SS electrodes were subjected to potential steps from open circuit potential to different potential values around the oxidation peak attributed to phenol oxidation (peak at $1.9 \mathrm{~V}$ ).

Figure 2 shows chronoamperometric curves for poly- 

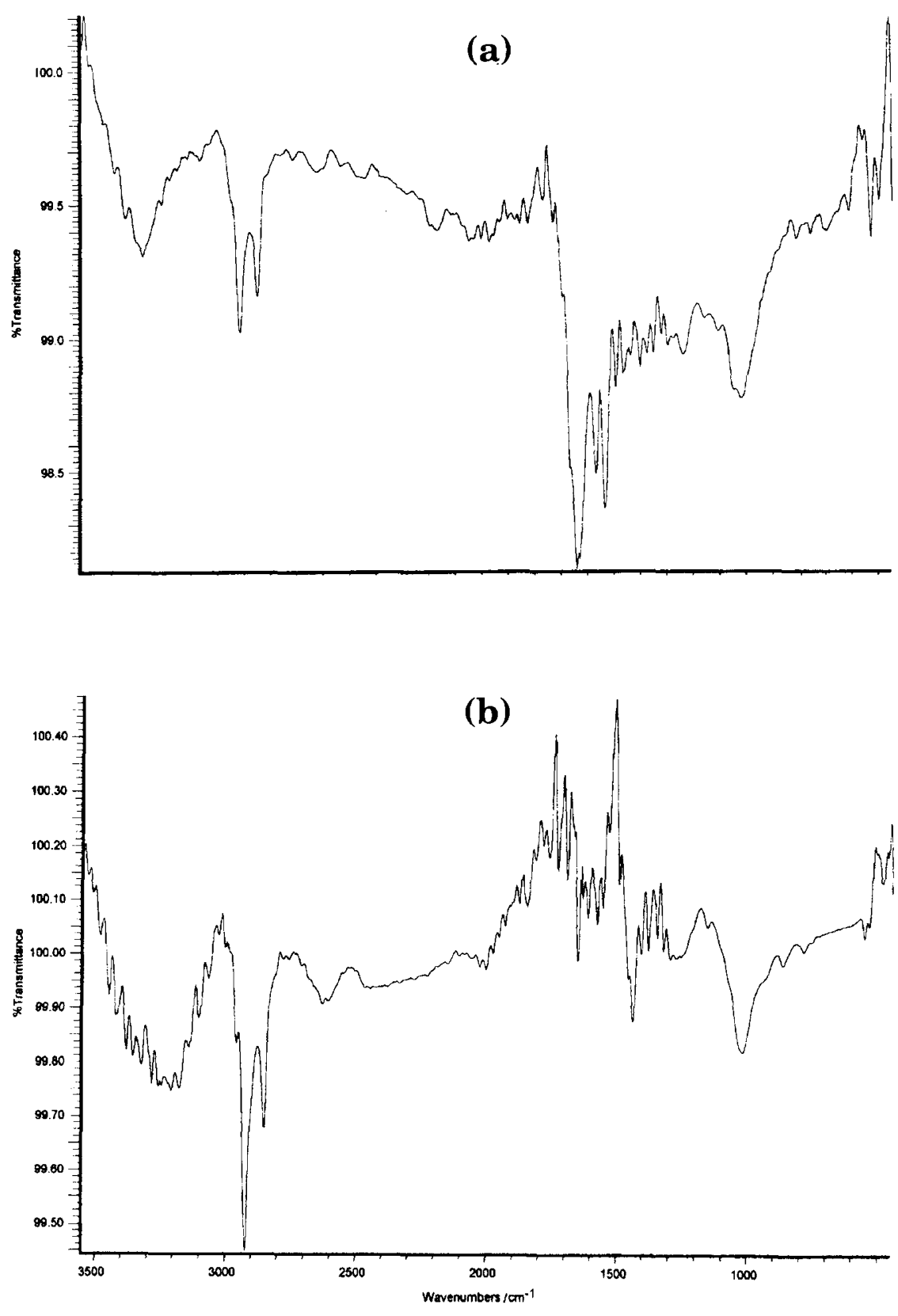

Figure 4. FTIR-ATR spectrum of polymer film created on both electrodes by electrooxidation of $6 \times 10^{-2} \mathrm{M}$ phenol in $0.1 \mathrm{M}$ $\mathrm{Na}_{2} \mathrm{CO}_{3}$ solution during $7 \mathrm{~min}$ at fixed potential of $1.9 \mathrm{~V}$. (a) on CS electrode. (b) on SS electrode.

mer formation obtained in $0.1 \mathrm{M}$ phenol $+0.1 \mathrm{M} \mathrm{Na}_{2} \mathrm{CO}_{3}$ solutions for different potentials, for CS electrode. In this figure, chronoamperometric curves for a potential of $1.6 \mathrm{~V}$ are presented because at this potential phenol oxidation starts. At all potentials, fast decrease in the current with the time (at very short time) was observed, probably because of the double layer charging. However, subsequent decay of the current with time depends on the applied potential. At potentials of $1.9 \mathrm{~V}$ or higher, fast decrease is followed by a hump and the electrode passivation is largely completed after a short period of time ( 1 to $2 \mathrm{~s}$ ). At potentials lower than $1.9 \mathrm{~V}$ the hump does not exist or is less evident, and passivation appears after a longer period of time. Integration of these current transients shows that the charge required for CS electrode passivation increases from 22.7 to $34.6 \mathrm{mC} \mathrm{cm}-2$ for potentials of 2.1 and $1.95 \mathrm{~V}$, respectively (Table I). For SS electrode the chronoamperometric curves are very similar and charges required for electrode passivation increase also from 19.3 to $28.4 \mathrm{mC} \mathrm{cm}^{-2}$ for potentials of 2.0 and $1.9 \mathrm{~V}$, respectively (Table II).

The small and steady current obtained at longer time (more than $13 \mathrm{~s}$ ), increases with positive potential, associated with the beginning of a slow oxidation of the polymeric film.

Figure 3 shows chroamperometric curves for CS electrodes and different phenol concentrations at a constant potential of $2.0 \mathrm{~V}$. As can be observed, the current decay profiles depend on phenol concentration. For smaller concentrations, the hump in the current and the electrode passivation appear at shorter time. Charge required to passivation decreases with phenol concentra- 
tion, as shown in Table I.

Similar behavior was observed with SS electrodes. Table II shows charges required for electrode passivation.

Film thickness was calculated from chronoamperometric curves and values between 0.10 and $0.20 \mu \mathrm{m}$ were obtained, close to those found by other authors. In ref 8 and 9 the films formed on Pt electrodes are smaller: $0.025 \mu \mathrm{m}$ for $\mathrm{Pt}$ in $\mathrm{NaOH}+2,6$-dimethylphenol aqueous solutions ${ }^{9}$ or approximately $0.01 \mu \mathrm{m}$ for tetramethylammonium phenoxide acetonitrile solutions. ${ }^{8}$ In ref 11 to 13 and 18 , in phenol $+\mathrm{KOH}$ solutions, coating film thickness lower than $1 \mu \mathrm{m}$ were obtained on mild steel electrodes. However, by adding ammonium compounds or suitable aliphatic amines, films of thickness higher than $10 \mu \mathrm{m}$ were formed.

Decrease of passivation rate when the phenol concentration increases (Figure 3 ) has been observed by other authors (i.e., Gattrell et al. working with phenol monomer in sulphuric acid medium ${ }^{6}$ and Glarum et al. which phenol derivatives in alkaline medium ${ }^{9}$ ). Gattrel et al. proposed a model in which the main products of the phenol oxidation are oligomers/polymers with high molecular weight that remain near the electrode surface. This product appears to be the initial material and that after its oxidation forms the immobilized and unreactive layer on the electrode surface that inhibits further reactions. Increase of phenol concentration in solution produces a higher phenol oxidation instead of oxidation of oligomers/polymers with high molecular weight material. Therefore, increase in phenol concentration increases the charge required for electrode passivation.

\section{IR Spectroscopy}

Figure 4 shows the FTIR-ATR spectra of the film produced by electrooxidation of $6 \times 10^{-2} \mathrm{M}$ phenol in carbonate medium for carbon and SS electrodes. For polymeric film formation, the electrode was subjected to one potential sweep from -0.5 to $1.9 \mathrm{~V}$ in this solution, and maintained at this final potential for $7 \mathrm{~min}$.

To assign the IR bands obtained for the polymeric film of Figure 4, the spectra of phenol monomer ${ }^{23}$ and polymeric film obtained on $\mathrm{Pt}$ electrodes in carbonate medium ${ }^{15}$ are used. The characteristic bands observed in Figure 4 are:

i) Two bands (weak) associated with the in-plane and out-of-plane aromatic ring deformation vibrations are observed in the $475-550 \mathrm{~cm}^{-1}$ region. ${ }^{24}$

ii) Several bands appear associated with aromatic outof-plane $\mathrm{C}-\mathrm{H}$ deformation vibration in the $700-850$ $\mathrm{cm}^{-1}$ region. ${ }^{24}$

iii) In the 900 to $1150 \mathrm{~cm}^{-1}$ spectral region, a broad and unresolved band associated with ether $\mathrm{C}-\mathrm{O}$ symmetric and asymmetric stretching vibration $(=\mathrm{C}-\mathrm{O}-\mathrm{C}=$ ring) ${ }^{24}$ is observed in both spectra.

iv) Two bands associated with the interaction of $\mathrm{O}-\mathrm{H}$ deformation and $\mathrm{C}^{-} \mathrm{O}$ stretching vibrations are observed in the $1260-1350 \mathrm{~cm}^{-1}$ region. ${ }^{24}$

v) At 1400 to $1650 \mathrm{~cm}^{-1}$ several bands appear associated with the aromatic carbon--carbon stretching vibration. ${ }^{24}$

vi) In the spectral region between 2800 to $3000 \mathrm{~cm}^{-1}$, several bands appear associated with aromatic $\mathrm{C}-\mathrm{H}$ stretching vibration. In the monomer spectrum,

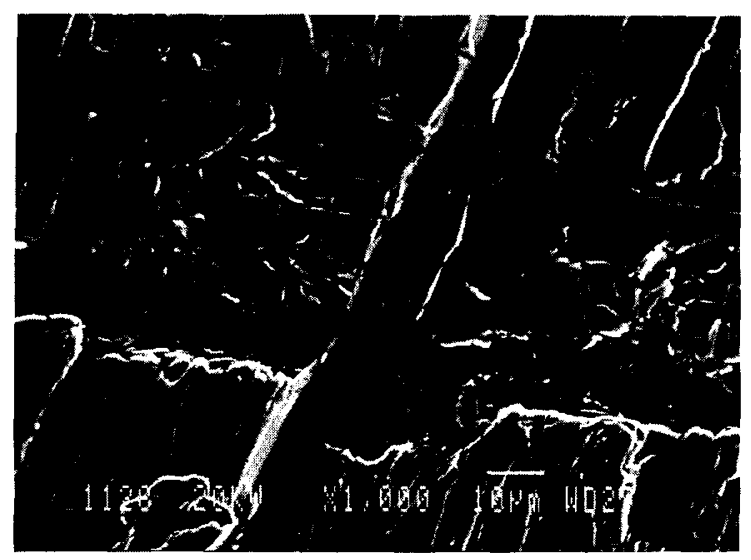

(a)

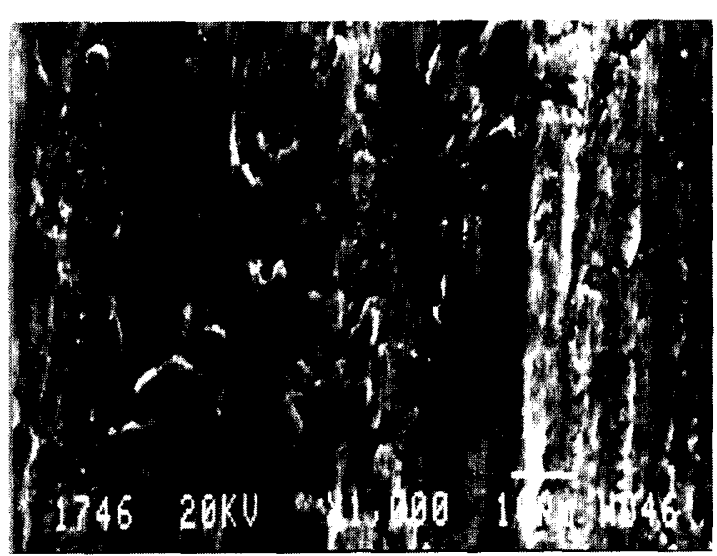

(b)

Figure 5. Scanning electron micrographs of: (a) bare CS electrode. (b) polymeric film formed on CS $0.1 \mathrm{M} \mathrm{Na}_{2} \mathrm{CO}_{3}+6 \times 10^{-2} \mathrm{M}$ phenol solution.

these bands are very small and they are not characteristics. Three well defined peaks are observed, as might be expected for multisubstituted benzenes (monosubstituted benzenes usually exhibit more peaks). ${ }^{24}$

vii) A broad band at $3300 \mathrm{~cm}^{-1}$ is also observed in both spectra attributed to the $\mathrm{O}-\mathrm{H}$ stretching vibration. ${ }^{24}$ A large decrease in the intensity of this band is observed compare to phenol monomer spectrum, ${ }^{23}$ associated with ether bond formation in the polymeric chain.

Other authors have obtained polymeric films by electropolymerization of phenol and its derivatives. Reference ${ }^{5}$ shows a transmission IR spectrum onto $\mathrm{KBr}$ disks of polyphenol obtained on platinum by anodic polarisation during the second time in sulphuric acid medium. Some remarkable differences appear between this and our spectra.

i) In ref 5 , the band at $3374 \mathrm{~cm}^{-1}$ associated to $\mathrm{O}-\mathrm{H}$ stretching vibration is very strong.

ii) The strong band does not appear in $900-1150 \mathrm{~cm}^{-1}$ region, associated with ether $\mathrm{C}-\mathrm{O}$ symmetric and asymmetric stretching vibrations $(=\mathrm{C}-\mathrm{O}-\mathrm{C}=$ ring $)$.

Conversely, reference ${ }^{10}$ shows a transmission spectra of the polymeric films obtained on Pt electrode, in $\mathrm{KOH}$ solutions, by anodic polarization, dissolved in chloroform 
and evaporated on a $\mathrm{NaCl}$ window. A broad and unresolved absorption band between $900-1050 \mathrm{~cm}^{-1}$ appears, ascribed to ether vibration.

On iron electrodes in oxalic acid solution with phenol derivatives, ${ }^{18}$ a coating consist of an inner Fe-oxalate and an outer polymer layer is obtained. The IR reflectance spectrum does not show bands associated with ether linked in the polymeric film.

Thus, $\mathrm{pH}$ effect exists in the electropolymerization mechanism of phenol. $\mathrm{C}-\mathrm{C}$ bonds are mainly formed in acid medium and $\mathrm{C}-\mathrm{O}$ bonds in alkaline medium.

From the ATR-IR spectrum, it may be concluded that the polymeric film formed on both steel electrodes obtained by us, maintains aromatic character and contains ether-linked rings.

\section{SEM Results}

To obtain information on the surface morphology of the polymeric films obtained on CS and SS electrodes, SEM was used. Figure 5a shows the SEM microphotograph of bare CS electrode. Figure $5 b$ shows the SEM microphotograph of CS electrode coated with the polymeric film. CS surface is covered by the polymer in spite roughness of the bare surface. In Figure $5 \mathrm{~b}$ the polymeric film surface shows a scaly aspect. A polymer with similar surface morphology is obtained on SS electrodes. This morphology is different from that obtained on platinum electrode in which different regions of homogeneous films separated by small wrinkles are obtained. ${ }^{15}$

\section{CONCLUSIONS}

From voltammetric and potentiostatic current transients results, it may be concluded:

i) The electrooxidation of phenol in carbonate medium on CS and SS electrodes causes the formation of a passivating film. This passivating film avoids further phenol oxidation and partially inhibits the electrode metal oxidation.

ii) The passivation rate of the electrodes decreases when phenol concentration increases or when the upper potential limit of the potential step decreases.

From the spectroscopic results it may be concluded:

i) The IR spectra of the polymeric films show characteristic bands of aromatic $\mathrm{C}-\mathrm{H}$ stretching vibration and aromatic $\mathrm{C}=\mathrm{C}$ stretching vibration. These bands permit a proposal that polymeric films created in carbonate medium maintain aromatic character.

ii) The intensity of the band associated to $\mathrm{O}-\mathrm{H}$ stretching vibration decreases in the IR spectrum. A band of ether $=\mathrm{C}-\mathrm{O}-\mathrm{C}=$ stretching vibration appears. The polymeric film thus contains ether-linked rings.

Acknowledgments. The authors thank the Dirección General de Enseñanza Superior e Investigación Científica (PB 97-0130) and Generalitat Valenciana (GV1159/93) and (AE 97-2) for the financial support.

\section{REFERENCES}

1. J. Wang, S. P. Chen, and M. S. Lin, J. Electroanal. Chem., 273, 231 (1989).

2. F. Bruno, M. C. Phan, and J. E. Dubois, Electrochim. Acta, 22, 451 (1977).

3. P. Mourcel, M. C. Phan, P. C. Lacaze, and J. E. Dubois, J. Electroanal.Chem., 145, 467 (1983) .

4. M. Delamar, M. Chemini, and J. E. Dubois, J. Electroanal Chem., 169, 145 (1984).

5. M. Gattrell and D. W. Kirk, J. Electrochem. Soc., 139, 2736 (1992) .

6. M. Gattrell and D. W. Kirk, J. Electrochem. Soc., 140,903 (1993).

7. M. Gattrell and D. W. Kirk, Can. J. Chem. Eng., 68, 997 (1990) .

8. R. L. McCarley, R. E. Thomas, E. A. Irene, and R. W. Murray, J. Electroanal. Chem., 290, 79 (1990) .

9. S. H. Glarum and J. H. Marshall, J. Electrochem. Soc., 132 , $2939(1985)$

10. S. H. Glarum, J. H. Marshall, M. Y. Hellman, and G. N. Taylor, J. Electrochem. Soc., 134, 81 (1987).

11. G. Mengoli and M. M. Musiani, J. Electrochem. Soc., 134, 643 c (1987).

12. G. Mengoli, M. M. Musiani, and F. Furlanetto, J. Electrochem. Soc., 137, 162 (1990).

13. M. Fleischman, I. R. Hill, G. Mengoli, and M. M. Musiani, Electrochim. Acta, 28, 1545 (1983).

14. G. Mengoli, P. Bianco, S. Daolio, and M. T. Munari, J. Electrochem. Soc., 128, 2276 (1981).

15. R. Lapuente, F. Cases, P. Garcés, E. Morallón, and J. L. Vázquez, J. Electroanal. Chem., 451 , 163 (1998).

16. I. Sekine, K. Kohara, T. Sugiyama, and M. Yuasa, J. Electrochem. Soc., 139, 3090 (1992).

17. M. M. Musiani, F. Furlanetto, P. Guerriero, and J. Heitbaum, J. Appl. Electrochem., 23, 1069 (1993).

18. G. Mengoli and M. M. Musiani, Electrochim. Acta, 31 ,201 (1986).

19. S. Tag, M.F. Ahmed, and S. Sankarapapavinasam, J. Appl. Electrochem., 23, 247 (1993).

20. R. Bertoncello, F. Furlanetto, A. Glisenti, and M.M. Musiani, J. Electrochem. Soc., 142, 410 (1995).

21. M. C. Pham, P. C. Lacaze, P. Mourcell, and J. E. Dubois, J. Appl. Electrochem., 16, 393 (1986).

22. R. Lapuente, Tesis de Licenciatura, Universidad de Alicante, 1998.

23. Aldrich Condensed Phase Sample Library, Nicolet Instruments, 1988.

24. G. Socrates, "Infrared Characteristic Group Frequencies" John Wiley \& Sons, 1994. 